\title{
Clinical diagnostic value of spiral CT in invasive pulmonary fungal infection
}

\author{
JUNWEI WANG ${ }^{1}$, CHUANYU ZHANG ${ }^{1}$, JIZHENG LIN ${ }^{1}$, LIANG ZHANG ${ }^{1}$, \\ $\mathrm{JIE} \mathrm{LI}^{1}$ and FANGFANG YANG ${ }^{2}$ \\ Departments of ${ }^{1}$ Radiology and ${ }^{2}$ Nephrology, The Affiliated Hospital of Qingdao University, \\ Qingdao, Shandong 266001, P.R. China
}

Received November 13, 2018; Accepted February 28, 2019

DOI: $10.3892 / \mathrm{etm} .2019 .7412$

\begin{abstract}
This study explored the diagnostic value of computed tomography (CT) in pulmonary fungal infection to provide a theoretical basis for the clinical diagnosis of pulmonary fungal infections. The clinical data of 82 suspected invasive fungal infection (IFI) patients admitted to the Department of Critical Care Medicine of The Affiliated Hospital of Qingdao University from January 2016 to May 2018 were retrospectively analyzed, and 64 of them were diagnosed with IFI by pathology and sputum culture. The CT results of the 82 patients were compared with the $\mathrm{X}$-ray results in order to analyze the diagnostic value of CT imaging. Taking pathological diagnosis as the gold standard, the number of true-negative, true-positive, false-negative and false-positive results in X-ray diagnosis were 13, 43, 21 and 5, respectively, while those in CT diagnosis were 11, 59, 5 and 7, respectively. The sensitivity, specificity, accuracy, positive coincidence rate, negative coincidence rate, misdiagnosis rate and missed diagnosis rate of CT in IFI were 92.18 , $61.11,85.37,89.39,68.75,38.89$ and $7.81 \%$, respectively, while those of X-ray in IFI were 67.19, 72.22, 68.29, 89.58, 38.24, 27.78 and $32.81 \%$, respectively. The sensitivity, accuracy and negative coincidence rate of CT in the diagnosis of IFI were significantly higher than those of $\mathrm{X}$-ray $(\mathrm{P}<0.05)$, with a sensitivity of $92.18 \%$, which indicates that CT has a higher diagnostic value in IFI. The results of CT combined with the basic condition of the patients can be used to initially diagnose pulmonary fungal infections, which is of high diagnostic value and can improve clinical treatment.
\end{abstract}

Correspondence to: Dr Fangfang Yang, Department of Nephrology, The Affiliated Hospital of Qingdao University, 16 Jiangsu Road, Qingdao, Shandong 266001, P.R. China

E-mail: r353uq@163.com

Key words: spiral computed tomography, invasive pulmonary fungal infection, diagnosis

\section{Introduction}

With the development of health care, the incidence of invasive fungal infections (IFIs) in the high risk and impaired immune-function population has been increasing (1). At present, it is believed that the main risk factors for IFI include neutropenia, malignant hematopathy, bone marrow transplantation, solid organ transplantation, severe burn, glucocorticoids, long-term intensive care, chemotherapy, HIV infection, invasive medical procedures and new immunosuppression agents (2-4). The patients in ICU have the characteristics of long-term bed rest, complicated basic diseases and low immune system, which create the conditions for the breeding and implantation of fungi, leading to an increasing number of pulmonary fungal infections (5-7). The early diagnosis of IFI, especially pulmonary fungal infection, remains a challenge in medical research (8). Invasive pulmonary aspergillosis (IPA) is the most common type in fungal pneumonia $(9,10)$. At present, the diagnosis of IFI relies on complex microbiological methods $(11,12)$, including culture and molecular identification.

Multi-slice spiral computed tomography (CT), a widely used imaging method in recent years, is reported to have achieved good results in the differential diagnosis of complicated pulmonary lesions by many scholars (13). Studies have also shown that CT is an important tool for the early diagnosis of pulmonary infection in immunocompromised hosts (14). The density and spatial resolution of multi-slice spiral CT have been greatly developed and unified. Its thin layer scan not only shows the details and changes of lung tissue more clearly, but also shows clearly all kinds of pathological changes, reconstructs images of any plane, such as axial, sagittal and coronal plane, and provides arbitrary section images by adjusting to different directions, allowing us to better understand the details of the lesion and the space anatomical relationship (15).

The purpose of this study was to investigate the diagnostic value of 64-slice spiral CT in invasive pulmonary fungal infection, and provide a reference for improving the treatment rate and prognosis of patients with pulmonary fungal infection.

\section{Patients and methods}

General information. The clinical data of 82 suspected IFI patients treated in the Department of Critical Care Medicine 
Table I. General information [n (\%)].

\begin{tabular}{lc}
\hline Clinical data & Cases $(\mathrm{n}=82)$ \\
\hline Sex & \\
Male & $51(62.20)$ \\
Female & $31(37.80)$ \\
Age (years) & \\
$\geq 40$ & $39(47.56)$ \\
$<40$ & $43(52.44)$ \\
BMI (kg/m²) & \\
$\geq 21$ & $37(45.12)$ \\
$<21$ & $45(54.88)$ \\
Smoking & \\
Yes & $59(71.95)$ \\
No & $23(28.05)$ \\
Hormone administration & \\
Yes & $48(58.54)$ \\
No & $34(41.46)$ \\
Severe disease classification & \\
Hematologic tumor & $21(25.61)$ \\
Immunosuppression therapy & $72(51.22)$ \\
Kidney transplantation & $7(8.54)$ \\
Solid tumor chemotherapy & \\
\hline
\end{tabular}

in The Affiliated Hospital of Qingdao University (Qingdao, China) from January 2016 to May 2018 were retrospectively analyzed, including 51 males and 31 females, with an average age of $40.59 \pm 10.34$ years. There were 21 patients with hematologic tumors, 42 patients treated with immunosuppression agents, 7 patients treated with kidney transplantation and 12 patients treated with solid tumor chemotherapy. The main clinical symptoms were cough and expectoration, and some patients presented with chest pain, hemoptysis, and fever. All patients underwent X-ray and spiral CT examinations during hospitalization. Finally, 64 patients were diagnosed with IFI by pathology and sputum culture. Inclusion criteria: suspected patients with clinical symptoms consistent with IFI. Exclusion criteria: patients with other severe infections; patients with allergies to CT contrast agents; patients with short-term expected death; patients previously using antifungal drugs. The patients and their families agreed to participate in tis study and signed an informed consent. The study was approved by the Ethics Committee of The Affiliated Hospital of Qingdao University. The general information of the patients is shown in Table I.

Detection methods. Sixty-four-slice spiral CT was performed using a CT scanner obtained from GE Healthcare (Chicago, IL, USA). Patients were placed in supine position and scanned with conventional plain scan from lung apex to lung bottom (voltage, $120 \mathrm{kV}$; current, 200-250 mA; thick section, $5 \mathrm{~mm}$; section interval, $5 \mathrm{~mm}$ ). If necessary, thinning and reconstruction were performed as needed, and enhanced examination was performed. The lesion location was scanned at a thickness of $2 \mathrm{~mm}$ and the section interval was $2 \mathrm{~mm}$. Mediastinal and

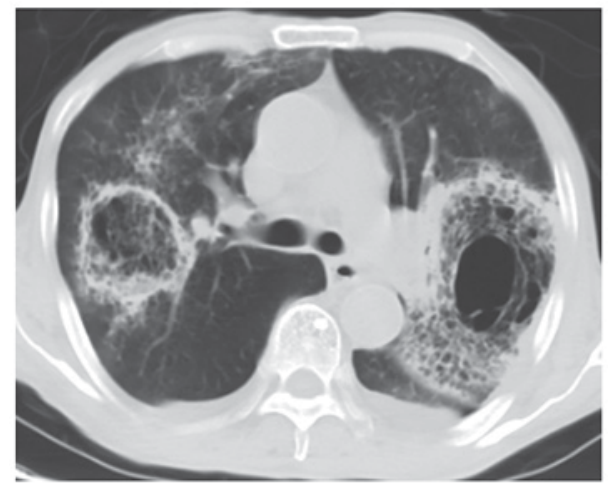

Figure 1. CT image of lung with pulmonary mucormycosis. The bilateral lungs show a symmetrical distribution of glass-like shadows with blurry boundaries and patchy solid shadows. CT, computed tomography.

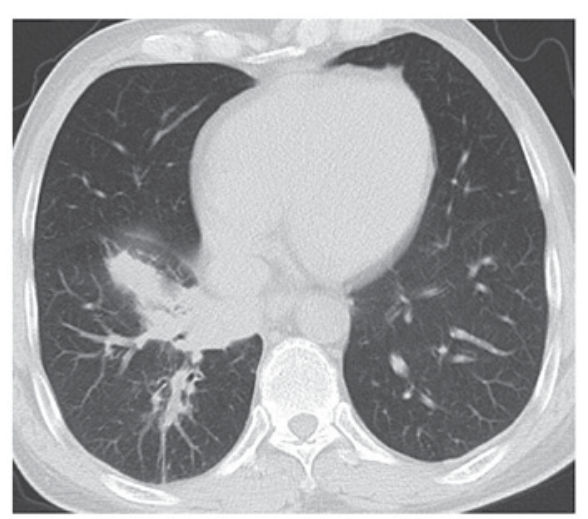

Figure 2. CT image of lung with pulmonary Candida infection. There are many nodules with different sizes and clear boundaries, accompanied by consolidation or tree-in-bud sign or ground-glass nodules, and there are halo signs around some nodules. CT, computed tomography.

pulmonary windows were used to observe the size, number, location, shape, density, boundary, surrounding structure and other imaging findings of lesions. The bedside chest radiography (X-ray) was performed by 12A radiographic device (Toshiba Medical Systems Corp., Tokyo, Japan), and chest radiography by GE Revolution XQ/i (GE Healthcare). All patients were examined in prone position and scans were carried out 2-3 times.

Outcome measures. Taking the results of pathology and sputum culture as the gold standard, the imaging features of CT in confirmed patients were analyzed and the sensitivity, specificity, accuracy, positive coincidence rate, negative coincidence rate, misdiagnosis rate and missed diagnosis rate of $\mathrm{X}$-ray and CT in IFI were calculated.

Statistical analysis. SPSS 17.0 statistical software (Shanghai Cabit Information Technology Co., Ltd., Shanghai, China) was used for analysis. t-test was used for the measurement data. $\mathrm{P}<0.05$ was considered to indicate a statistically significant difference.

\section{Results}

Spiral CTfindings in 64 patients. Among 64 confirmed patients, there were 14 cases of pulmonary mucormycosis, 15 cases of 


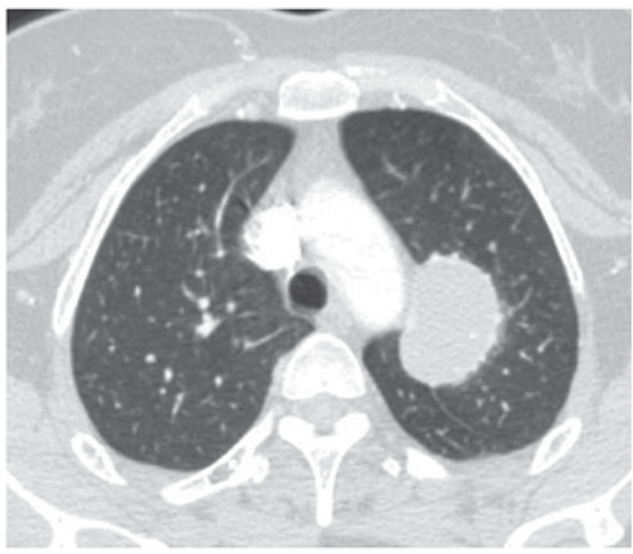

Figure 3. CT image of lung with pulmonary Aspergillus infection. The inside cavity is characterized by the inhomogeneous density of the sphere, rough edge, and the meniscus sign in meniscus radiolucent area of the cavity wall. CT, computed tomography.

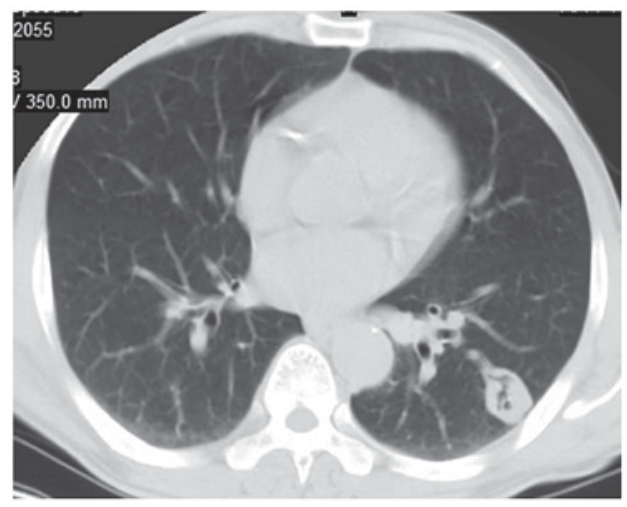

Figure 4. CT image of lung with pulmonary Cryptococcus infection. There are single or multiple nodules or masses with multiple distribution and clear boundaries in most of them, and the lesions are distributed as lobes or segments. CT, computed tomography.

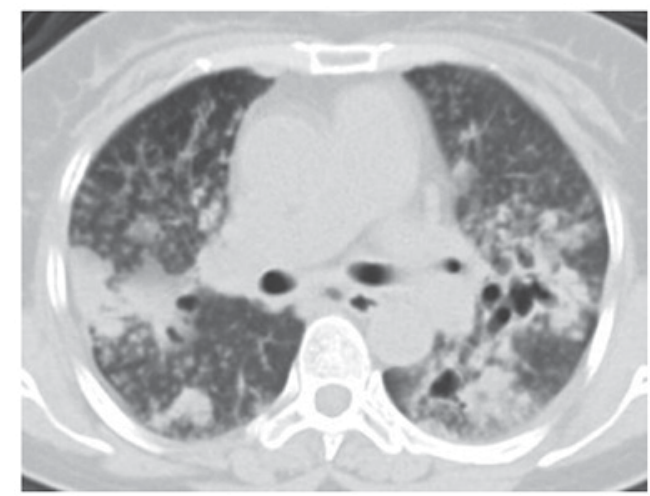

Figure 5. CT image of lung with pulmonary histoplasmosis. There is miliary pulmonary infiltration, cavitation formation and hilar lymphadenectasis, and significant hilar lymph nodes. In part of the lung apex appears a cavity with no obvious specific manifestations. CT, computed tomography.

pulmonary Candida infection, 20 cases of pulmonary Aspergillus infection, 13 cases of pulmonary Cryptococcus infection and 2 cases of pulmonary histoplasmosis. Representative CT scans are shown in Figs. 1-5.
Table II. X-ray diagnosis.

\begin{tabular}{lccc}
\hline & \multicolumn{2}{c}{ Pathological diagnosis } & \\
\cline { 2 - 3 } X-ray diagnosis & Negative & Positive & Total \\
\hline Negative & 13 & 21 & 34 \\
Positive & 5 & 43 & 48 \\
Total & 18 & 64 & 82 \\
\hline
\end{tabular}

Table III. CT diagnosis.

\begin{tabular}{lccc}
\hline & \multicolumn{2}{c}{ Pathological diagnosis } & \\
\cline { 2 - 3 } CT diagnosis & Negative & Positive & Total \\
\hline Negative & 11 & 5 & 16 \\
Positive & 7 & 59 & 66 \\
Total & 18 & 64 & 82 \\
\hline
\end{tabular}

CT, computed tomography.

Table IV. Comparison of the diagnostic value of X-ray and CT.

\begin{tabular}{lcccr}
\hline & $\begin{array}{c}\text { X-ray } \\
(\mathrm{n}=82) \\
(\%)\end{array}$ & $\begin{array}{c}\mathrm{CT} \\
(\mathrm{n}=82) \\
(\%)\end{array}$ & $\mathrm{t}$ & P-value \\
Diagnosis & 67.19 & 92.18 & 12.36 & $<0.001$ \\
Sensitivity & 72.22 & 61.11 & 0.500 & 0.480 \\
Specificity & 68.29 & 85.37 & 6.713 & $<0.050$ \\
Accuracy & 89.58 & 89.39 & 0.001 & 0.974 \\
Positive coincidence rate & 38.24 & 68.75 & 18.68 & $<0.001$ \\
Negative coincidence rate & 37.78 & 38.89 & 0.500 & 0.480 \\
Misdiagnosis rate & 32.81 & 7.81 & 12.36 & $<0.001$ \\
Missed diagnosis rate & & & &
\end{tabular}

CT, computed tomography.

$X$-ray and CT diagnosis. Taking pathological diagnosis as the gold standard, the number of true-negative, true-positive, false-negative and false-positive results in X-ray diagnosis were 13,43, 21 and 5, respectively, while those in CT diagnosis were $11,59,5$ and 7, respectively (Tables II and III).

Comparison of diagnostic value of X-ray and CT. The sensitivity, specificity, accuracy, positive coincidence rate, negative coincidence rate, misdiagnosis rate and missed diagnosis rate of X-ray in IFI were $67.19,72.22,68.29,89.58,38.24$, 27.78 and $32.81 \%$, respectively, while those of CT in IFI were $92.18,61.11,85.37,89.39,68.75,38.89$ and $7.81 \%$, respectively. The sensitivity, accuracy and negative coincidence rate of CT in the diagnosis of IFI were significantly higher than those of $\mathrm{X}$-ray $(\mathrm{P}<0.05)$, with a sensitivity of $92.18 \%$, which indicates that CT had a high diagnostic value in IFI (Table IV). 


\section{Discussion}

In recent years, with the widespread use of antibiotics, hormones and immunosuppression agents, the number of IFIs has increased (16). However, because of the lack of clinical specificity of IFI, the condition is easily covered up by the primary disease, resulting in delay of the treatment. This is the reason why the highest mortality rate can reach $80 \%$ (17). IFI has become one of the leading causes of death in patients with solid organ transplantation and hematological malignancy $(18,19)$. Therefore, the way to make accurate and in time diagnosis of IFI is an urgent issue to be addressed. At present, the gold standard for the diagnosis of IFI is pathological biopsy, but it is not accepted by patients and doctors because of the trauma caused. The sensitivity and specificity of traditional methods, such as respiratory sputum culture are only $\sim 50 \%$ (20). Studies have shown that CT plays an important role in the diagnosis of fungal infections (21). CT has also been reported to have higher spatial and density resolution than X-ray, and to make good imaging observation of the basic pathological changes in IFI (22). In the diagnosis of pulmonary fungal infection, CT is expressed as scattered nodules, masses or patchy focis, and other signs, such as halo. Meniscus signs may also appear, and the occurrence of one or both signs at the same time is common (23). It has also been reported that subpleural wedge-shaped consolidation may occur in invasive pulmonary Aspergillus infection imaging (24).

In the present study, with pathological diagnosis as the gold standard, the diagnostic value of X-ray and CT in IFI was compared and the imaging features of CT diagnosis were analyzed. The results showed that the sensitivity, accuracy and negative coincidence rate of $\mathrm{CT}$ in the diagnosis of IFI were significantly higher than those of $\mathrm{X}$-ray $(\mathrm{P}<0.05)$, with a sensitivity of $92.18 \%$, which indicates that CT has a high diagnostic value in IFI. By analyzing the causes of misdiagnosis and missed diagnosis, it has been found that 6 out of 7 cases misdiagnosed with CT are caused by host factors. Although some studies have shown that the diagnosis of fungal infection has a greater dependence on host factors (11), we believe that misdiagnosis can also be caused by excessive interpretation of host factors in the misdiagnosis case analysis. When the causes of misdiagnosis and missed diagnosis of X-ray were analyzed, it was found that most of them were difficult to diagnose because of unclear imaging features. Therefore, we believe that when imaging cannot be judged, sputum culture or serum marker examination should be performed in time to further determine the diagnostic results. For example, when a patient has pulmonary artery embolism and incurable long-term pulmonary abscess, it can be considered as pulmonary mucormycosis infection. In the analysis of the imaging features of CT in this study, it was found that the multiple nodules and meniscus signs appeared more frequently in the collected cases, which coincided with the CT imaging features of IFI described in related literature (22). Some studies have suggested that the CT manifestations of pneumonia caused by different strains have their own characteristics (25). For example, meniscus sign is the characteristic of Aspergillus infection, and multiple nodule and small patch are the characteristics of Cryptococcus infection. In the diagnostic criteria developed by the European Organization for Cancer Therapy and Fungal Research Group, the meniscus sign and the cavity appearing in the consolidation are all characteristic manifestations of pulmonary fungal infection (26).

In conclusion, the diagnostic value of CT in IFI is higher than that of traditional X-ray, and CT has a better enhancement effect in many fungal areas infected with IFI. However, some lesions are difficult to distinguish, so it is necessary to make a comprehensive judgment and diagnosis by combining the clinical data and the medical history of the patients.

\section{Acknowledgements}

Not applicable.

\section{Funding}

No funding was received.

\section{Availability of data and materials}

The datasets used and/or analyzed during the present study are available from the corresponding author on reasonable request.

\section{Authors' contributions}

JW, JLi and FY were responsible for the CT result analysis. JLin and CZ recorded and analyzed the general data of patients. JLi, FY and LZ contributed to the statistical analysis. The final version was read and approved by all the authors.

\section{Ethics approval and consent to participate}

The study was approved by the Ethics Committee of the Affiliated Hospital of Qingdao University (Qingdao, China). Signed written informed consents were obtained from the patients or their guardians.

\section{Patient consent for publication}

Not applicable.

\section{Competing interests}

The authors declare that they have no competing interests.

\section{References}

1. Badiee P and Hashemizadeh Z: Opportunistic invasive fungal infections: Diagnosis \& clinical management. Indian J Med Res 139: 195-204, 2014

2. Ribes JA, Vanover-Sams CL and Baker DJ: Zygomycetes in human disease. Clin Microbiol Rev 13: 236-301, 2000.

3. Baddley JW: Clinical risk factors for invasive aspergillosis. Med Mycol 49 (Suppl 1): S7-S12, 2011.

4. Badiee P, Alborzi A and Farhoudi F: A case of Candida mediastinitis after dental extraction. J Infect Dev Ctries 5: 75-78, 2011.

5. Vandewoude KH, Blot SI, Depuydt P, Benoit D, Temmerman W, Colardyn F and Vogelaers D: Clinical relevance of Aspergillus isolation from respiratory tract samples in critically ill patients. Crit Care 10: R31, 2006.

6. Garnacho-Montero J, Amaya-Villar R, Ortiz-Leyba C, León C, Alvarez-Lerma F, Nolla-Salas J, Iruretagoyena JR and Barcenilla F: Isolation of Aspergillus spp. from the respiratory tract in critically ill patients: Risk factors, clinical presentation and outcome. Crit Care 9: R191-R199, 2005. 
7. Cornillet A, Camus C, Nimubona S, Gandemer V, Tattevin P, Belleguic C, Chevrier S, Meunier C, Lebert C, Aupée M, et al: Comparison of epidemiological, clinical, and biological features of invasive aspergillosis in neutropenic and nonneutropenic patients: A 6-year survey. Clin Infect Dis 43: 577-584, 2006.

8. Georgiadou SP, Sipsas NV, Marom EM and Kontoyiannis DP The diagnostic value of halo and reversed halo signs for invasive mold infections in compromised hosts. Clin Infect Dis 52 : $1144-1155,2011$

9. Bruno C,Minniti S, Vassanelli A and Pozzi-Mucelli R: Comparison of CT features of Aspergillus and bacterial pneumonia in severely neutropenic patients. J Thorac Imaging 22: 160-165, 2007.

10. Escuissato DL, Gasparetto EL, Marchiori E, Rocha GM, Inoue C, Pasquini R and Müller NL: Pulmonary infections after bone marrow transplantation: high-resolution CT findings in 111 patients. AJR Am J Roentgenol 185: 608-615, 2005.

11. Hope WW, Walsh TJ and Denning DW: Laboratory diagnosis of invasive aspergillosis. Lancet Infect Dis 5: 609-622, 2005.

12. Yeo SF and Wong B: Current status of nonculture methods for diagnosis of invasive fungal infections. Clin Microbiol Rev 15: 465-484, 2002

13. Ma SH, Xu K, Xiao ZW, Wu M, Sun ZY, Wang ZX, Hu ZG, Dai X, Han MJ and Li YG: Peripheral lung cancer: Relationship between multi-slice spiral CT perfusion imaging and tumor angiogenesis and cyclin D1 expression. Clin Imaging 31: 165-177, 2007.

14. Caillot D, Couaillier JF, Bernard A, Casasnovas O, Denning DW, Mannone L, Lopez J, Couillault G, Piard F, Vagner O, et al: Increasing volume and changing characteristics of invasive pulmonary aspergillosis on sequential thoracic computed tomography scans in patients with neutropenia. J Clin Oncol 19: 253-259, 2001.

15. Fuchs T, Kachelriess M and Kalender WA: Technical advances in multi-slice spiral CT. Eur J Radiol 36: 69-73, 2000.

16. Yang WL, Cao J, Chen BY, Xie W, Dong LX, Wu YQ, Li JN and $\mathrm{Hu} \mathrm{ZD}$ : A preliminary study on the measurement of $(1,3)$ - $\beta$-D-glucan in bronchoalveolar lavage for the diagnosis of pulmonary fungal infections. Zhonghua Jie $\mathrm{He} \mathrm{He} \mathrm{Hu} \mathrm{Xi} \mathrm{Za}$ Zhi 35: 897-900, 2012 (In Chinese).

17. Lin SJ, Schranz J and Teutsch SM: Aspergillosis case - fatality rate: Systematic review of the literature. Clin Infect Dis 32: 358-366, 2001

18. Segal BH and Walsh TJ: Current approaches to diagnosis and treatment of invasive aspergillosis. Am J Respir Crit Care Med 173: 707-717, 2006.
19. Patterson TF: Advances and challenges in management of invasive mycoses. Lancet 366: 1013-1025, 2005.

20. Prasad A, Agarwal K, Deepak D and Atwal SS: Pulmonary Aspergillosis: What CT can offer before it is too late! J Clin Diagn Res 10: TE01-TE05, 2016.

21. Christe A, Lin MC, Yen AC, Hallett RL, Roychoudhury K, Schmitzberger F, Fleischmann D, Leung AN, Rubin GD, Vock $\mathrm{P}$, et al: CT patterns of fungal pulmonary infections of the lung: Comparison of standard-dose and simulated low-dose CT. Eur J Radiol 81: 2860-2866, 2012.

22. Park SY, Kim SH, Choi SH, Sung H, Kim MN, Woo JH, Kim YS, Park SK, Lee JH, Lee KH, et al: Clinical and radiological features of invasive pulmonary aspergillosis in transplant recipients and neutropenic patients. Transpl Infect Dis 12: 309-315, 2010.

23. Franquet T, Müller NL, Giménez A, Guembe P, de La Torre J and Bagué S: Spectrum of pulmonary aspergillosis: Histologic, clinical, and radiologic findings. Radiographics 21: 825-837, 2001.

24. Paulussen C, Hallsworth JE, Álvarez-Pérez S, Nierman WC, Hamill PG, Blain D, Rediers H and Lievens B: Ecology of aspergillosis: Insights into the pathogenic potency of Aspergillus fumigatus and some other Aspergillus species. Microb Biotechnol 10: 296-322, 2017

25. Demirkazik FB, Akin A, Uzun O, Akpinar MG and Ariyürek MO: CT findings in immunocompromised patients with pulmonary infections. Diagn Interv Radiol 14: 75-82, 2008.

26. De Pauw B, Walsh TJ, Donnelly JP, Stevens DA, Edwards JE, Calandra T, Pappas PG, Maertens J, Lortholary O, Kauffman CA, et al; European Organization for Research and Treatment of Cancer/Invasive Fungal Infections Cooperative Group; National Institute of Allergy and Infectious Diseases Mycoses Study Group (EORTC/MSG) Consensus Group: Revised definitions of invasive fungal disease from the European Organization for Research and Treatment of Cancer/Invasive Fungal Infections Cooperative Group and the National Institute of Allergy and Infectious Diseases Mycoses Study Group (EORTC/MSG) Consensus Group. Clin Infect Dis 46: 1813-1821, 2008.

This work is licensed under a Creative Commons Attribution-NonCommercial-NoDerivatives 4.0 International (CC BY-NC-ND 4.0) License. 